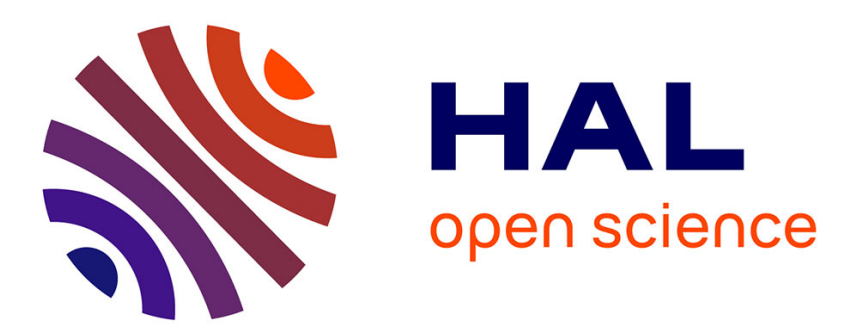

\title{
Etude du vieillissement d'éléments chauffants à haut-flux en graphite ou en composite carbone-carbone
}

\author{
H. Fuzellier, D. Guérard, K. Jaume, C. Oberlin
}

\section{To cite this version:}

H. Fuzellier, D. Guérard, K. Jaume, C. Oberlin. Etude du vieillissement d'éléments chauffants à haut-flux en graphite ou en composite carbone-carbone. Journal de Physique IV Proceedings, 1993, 03 (C9), pp.C9-889-C9-899. 10.1051/jp4:1993992 • jpa-00252434

\section{HAL Id: jpa-00252434 https://hal.science/jpa-00252434}

Submitted on 1 Jan 1993

HAL is a multi-disciplinary open access archive for the deposit and dissemination of scientific research documents, whether they are published or not. The documents may come from teaching and research institutions in France or abroad, or from public or private research centers.
L'archive ouverte pluridisciplinaire HAL, est destinée au dépôt et à la diffusion de documents scientifiques de niveau recherche, publiés ou non, émanant des établissements d'enseignement et de recherche français ou étrangers, des laboratoires publics ou privés. 


\title{
Etude du vieillissement d'éléments chauffants à haut-flux en graphite ou en composite carbone-carbone
}

\author{
H. Fuzellier( $\left.{ }^{1}\right)$, D. Guérard $\left({ }^{1}\right)$, R. Jaume $\left({ }^{2}\right)$ et C. Oberlin $\left({ }^{2}\right)$
}

$\left({ }^{1}\right)$ Laboratoire de Chimie du Solide Minéral, URA 158, Université de Nancy I, Boulevard des Aiguillettes - Entrée 5A, B.P. 239, 54506 Vandoeuvre-Lès-Nancy, France

$\left({ }^{2}\right)$ Electricité de France, Direction des Etudes et Recherches, Département Applications de l'Electricité dans l'Industrie, Les Renardières, Route de Sens, B.P. 1, 77250 Moret-SurLoing, France

\begin{abstract}
Résumé. - Pour réaliser des thermoplongeurs chauffants à haut flux, différents matériaux carbonés ont été mis en oeuvre : graphite polycristallin et composites $\mathrm{C} / \mathrm{C}$. Nous avons mis en évidence l'influence de certaines impuretés : silice, fer et calcium (combinées ou non) qui sont à l'origine d'une forte corrosion et qui doivent être éliminées. Pour limiter le transport du carbone dans l'appareillage, il convient d'utiliser une matrice carbonée graphitée à haute température : soit en ex-résine, soit en pyrocarbone.
\end{abstract}

\begin{abstract}
To realize highly efficient heating element operating at $1400{ }^{\circ} \mathrm{C}$ under nitrogen environment, with a specific power of $500 \mathrm{~W} / \mathrm{cm}^{2}$, various carbon materials have been progressively selected: polycrystalline graphite and carbon/carbon composites coated or not. Some drastic corrosion effects, due to impurities: silica, iron and calcium, have been underlined. To limit carbon transport in the reactor, the carbon matrix of the resistance must be graphitized at high temperature (pyrocarbon or carbonized resin).
\end{abstract}

\section{Introduction.}

Depuis quelques années, EDF entreprend des essais de faisabilité sur des résistances chauffantes haut-flux à base de carbone. Différents matériaux carbonés : graphite et composites carbone/carbone [1-4] revêtus ou non, sont développés avec plusieurs fabricants de matériaux.

Les essais menés dans les laboratoires d'Electricité de France aux Renardières ont pour but de choisir le matériau le plus performant pour une durée de vie maximale (plus de 5000 heures).

\section{Conditions d'essais.}

Les conditions d'essai peuvent se résumer de la façon suivante :

Les résistances (Fig. 1) sont montées dans une gaine étanche contenant de l'azote. L'ensemble est installé dans une chaudière (Fig. 2) dont le débit d'eau est réglé de façon à maintenir une température comprise entre 60 et $80^{\circ} \mathrm{C}$, la température de l'élément chauffant étant ainsi stabilisée à $1400^{\circ} \mathrm{C}$ pendant des durées variables : 500, 1000, 1500, 3000, 4000 et 5000 heures, voire au-delà. 


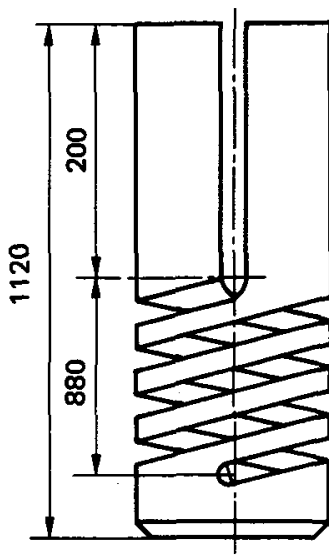

(a)

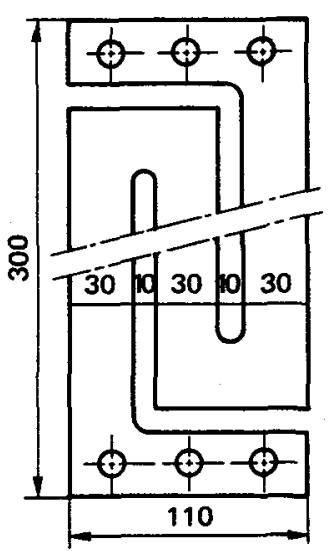

(b)

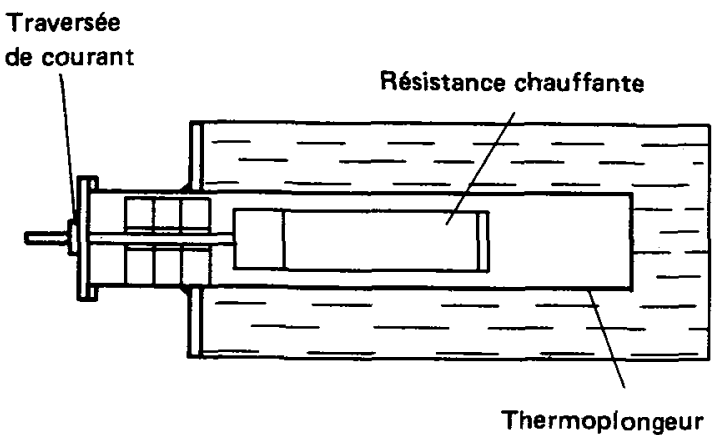

Fig. 2

Fig. 1

Fig. 1. - Schéma des résistances chauffantes : a) graphite; b) composite C/C.

Fig. 2. - Schéma du montage d'essai des résistances chauffantes.

\section{Résultats expérimentaux.}

Dans chaque série d'essais, des échantillons sont prélevés pour des examens approfondis au Laboratoire de Chimie du Solide Minéral de Nancy : étude macroscopique (lunette binoculaire), étude microscopique par microscopie électronique à balayage, par diffraction des rayons $\mathrm{X}$ et par microsonde électronique.

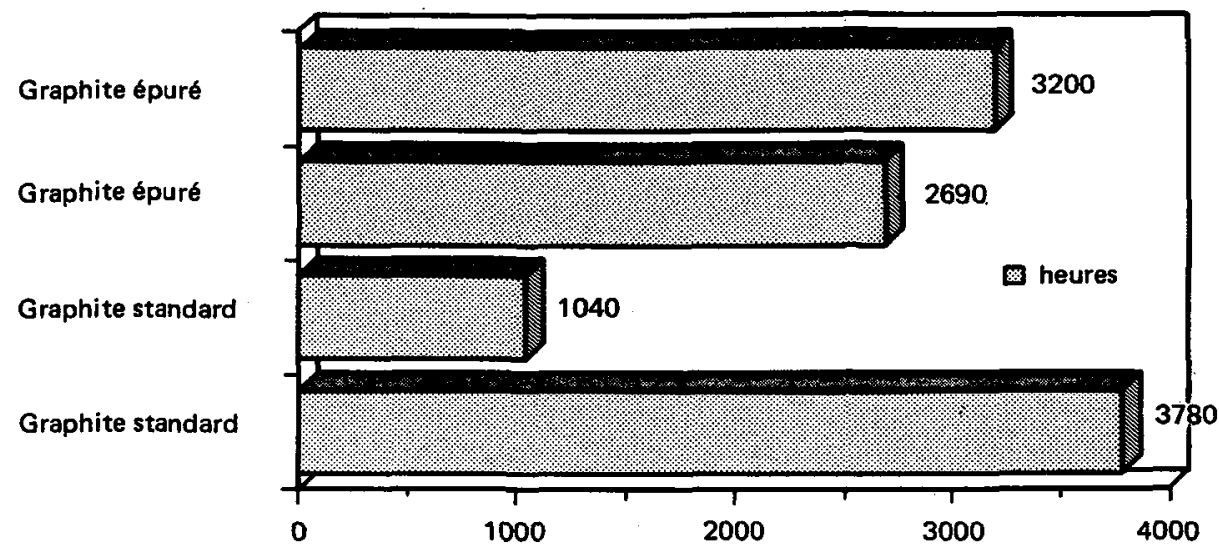

Fig. 3. - Durée de vie des résistances en graphite.

Sur les figures 3 et 4 sont reportées les durées de vie des différentes résistances carbonées. Celles-ci n'évoluent pas d'une manière simple et logique. La dispersion des durées de vie sur un même type de graphite (graphite standard) est liée à des conditions d'essais mal 


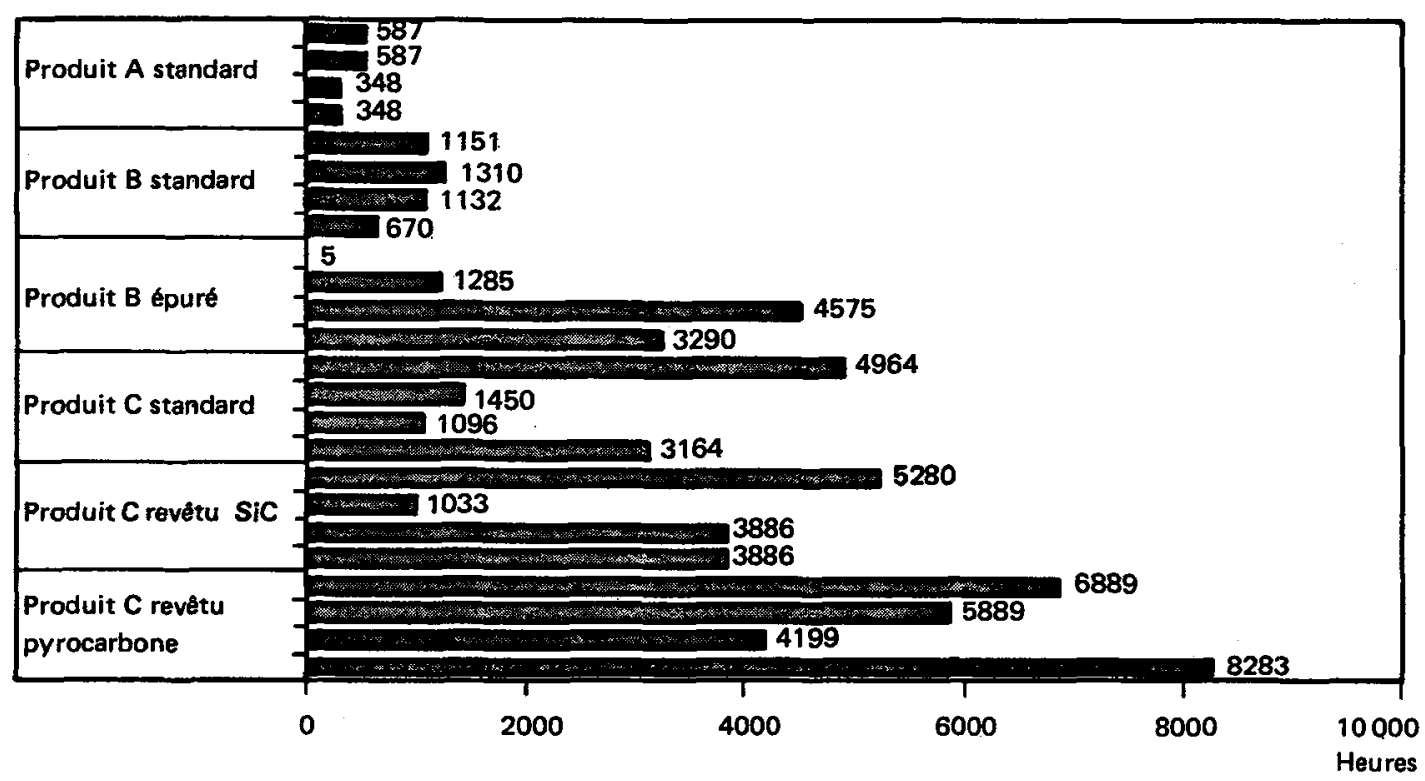

Fig. 4. - Durée de vie des résistances en composite $\mathrm{C} / \mathrm{C}$.

maîtrisées pour un des échantillons ; pour les produits à base de composites carbone-carbone, la dispersion est due à la difficulté de prélever des échantillons homogènes dans une plaque épaisse et de grandes dimensions. Parmi les nombreux paramètres intervenants, nous avons étudié, plus particulièrement, l'influence des impuretés contenues dans les matériaux.

3.1 INFLUENCE DE LA SILICE. - Les premières expériences mettent en oeuvre des résistances en graphite artificiel, standard ou épuré, et en composite carbone/carbone (Echantillon A). La durée de vie de ces dernières est très courte (quelques centaines d'heures : Fig. 4) et leur fonctionnement s'accompagne d'un important dépôt de carbone à l'intérieur de la gaine : environ $150 \mathrm{~g} / \mathrm{kg}$ de graphite en $3200 \mathrm{~h}$ ! Dans le cas du graphite artificiel, épuré ou non, le dépôt est moins important ( $17 \mathrm{~g} / \mathrm{kg}$ de graphite).

Par ailleurs, les résistances présentent des traces d'oxydation importante, bien que l'appareil soit sous atmosphère d'azote : apparition d'une porosité ouverte importante (Photos 1 et 2), bords des particules de graphite arrondis (Photo 3 ) et fibres de carbone corrodées et dégagées de la matrice carbonée présentant des piqûres profondes (Photos 4 à 8 ).

L'origine de cette oxydation provient en réalité d'un joint isolant, en fibres silicoaluminées, monté au niveau des amenées de courant. Lobservation et l'analyse de certaines de ces fibres au microscope électronique à balayage montrent qu'il ne subsiste plus qu'un squelette d'alumine pure dû à la volatilisation totale de $\mathrm{SiO}_{2}$ sous forme de $\mathrm{SiO}$ (Photo 9) ; ceci nous conduit à envisager les réactions suivantes :

$$
\begin{gathered}
\mathrm{SiO}_{2}, x \mathrm{Al}_{2} \mathrm{O}_{3}+\mathrm{C} \rightarrow x \mathrm{Al}_{2} \mathrm{O}_{3}+\mathrm{SiO}+\mathrm{CO} \\
\mathrm{SiO}+\mathrm{CO} \rightleftarrows \mathrm{SiO}_{2}+\mathrm{C} \\
2 \mathrm{CO} \rightleftarrows \mathrm{C}+\mathrm{CO}_{2}
\end{gathered}
$$

Ces trois réactions ont pour mérite d'expliquer nos observations : réduction et vaporisation 
de la silice sous forme de monoxyde de silicium volatil [5], apparition de monoxyde de carbone dans le réacteur qui, en présence de graphite porté à $1400{ }^{\circ} \mathrm{C}$ et d'une paroi maintenue à $80^{\circ} \mathrm{C}$ environ, fait intervenir l'équilibre de Boudouard et permet d'expliquer le transport du carbone dans la gaine.

Le carbone déposé (Photo 10) est constitué de petites particules (diamètre : 10 à $20 \mu \mathrm{m}$ ) présentant une bonne organisation 3D ; l'analyse du diffractogramme X du film carboné (Photo 11) permet d'évaluer son taux de graphitation à $60 \%$. Sur les extrémités de la résistance, zones plus froides, apparaissent des fibres de silice cristallisée (Photo 12).

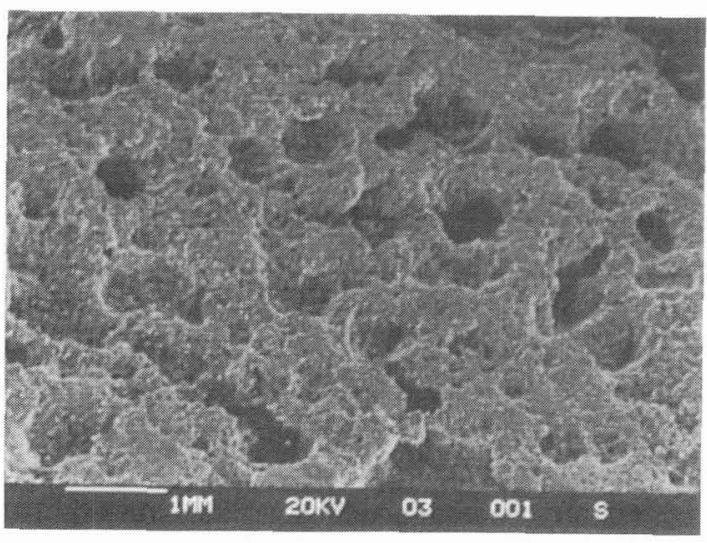

Photo 1

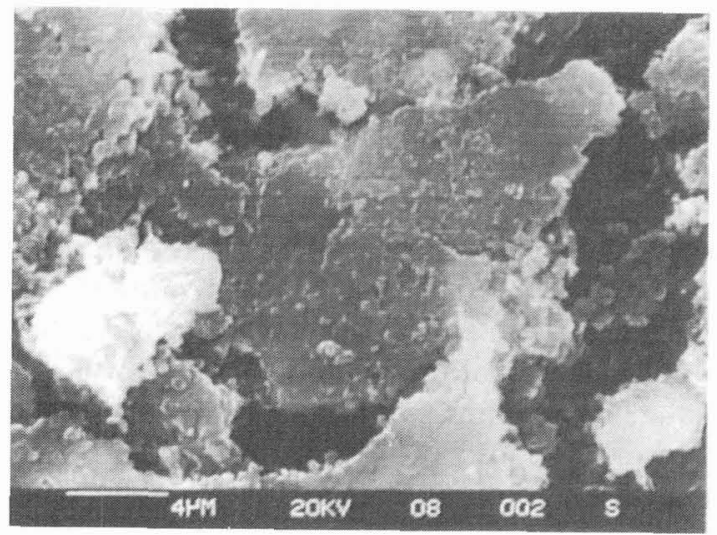

Photo 3

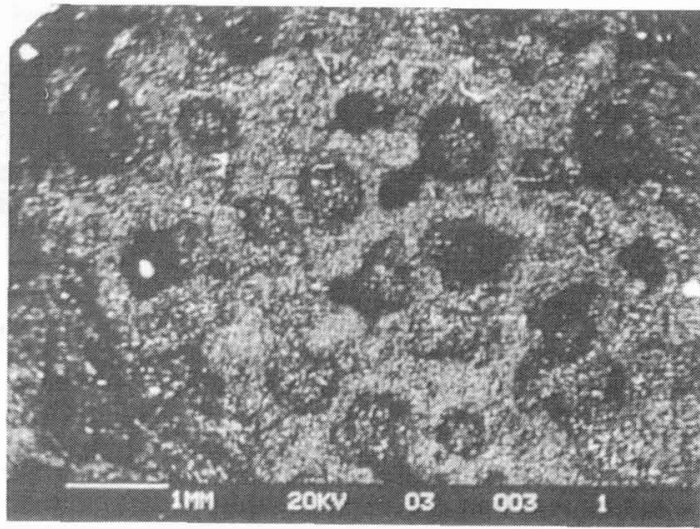

Photo 2

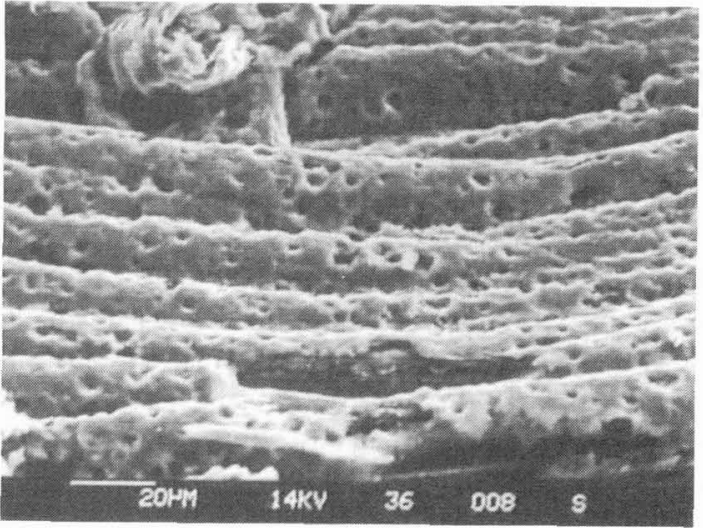

Photo 4

Photo 1. - Observation au MEB de la porosité superficielle (électrons secondaires).

Photo 2. - Observation au MEB de la porosité superficielle (électrons rétrodiffusés).

Photo 3. - Observation au MEB des bords arrondis des cristallites du graphite polycristallin après corrosion.

Photos 4-8. - Piqûres de corrosion des gaines en pyrocarbone enveloppant les fibres de carbone. 


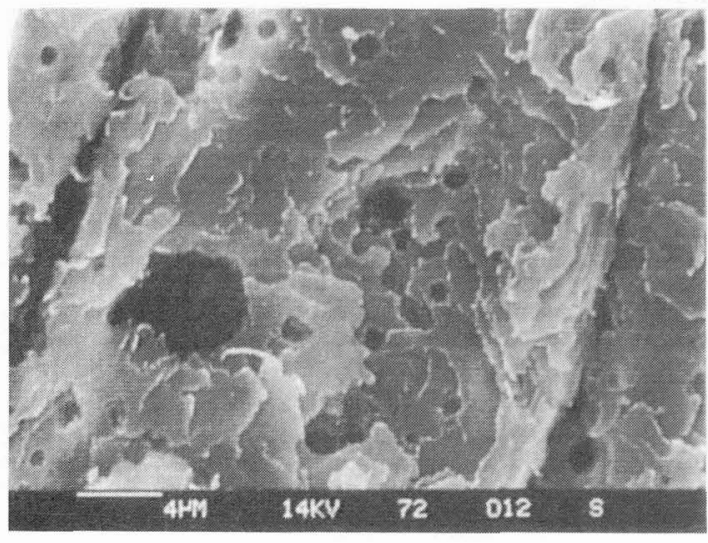

Photo 5

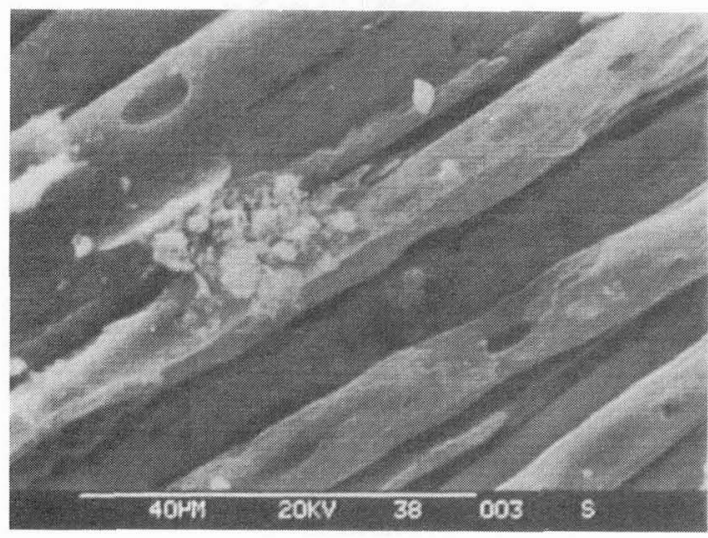

Photo 7

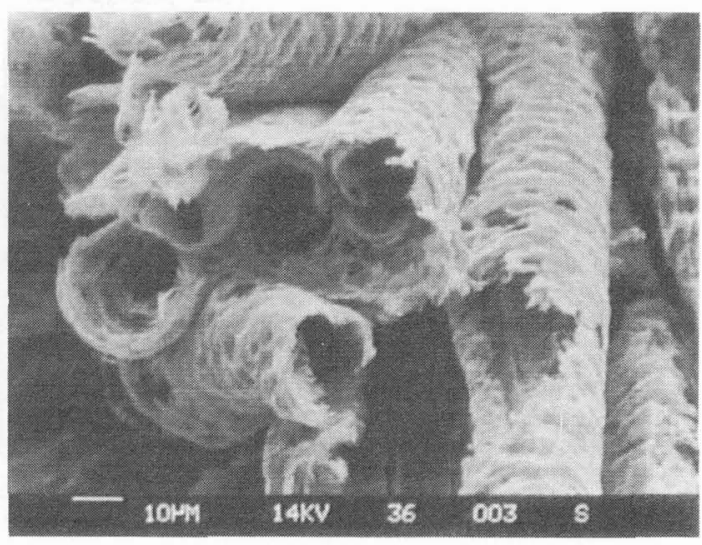

Photo 6

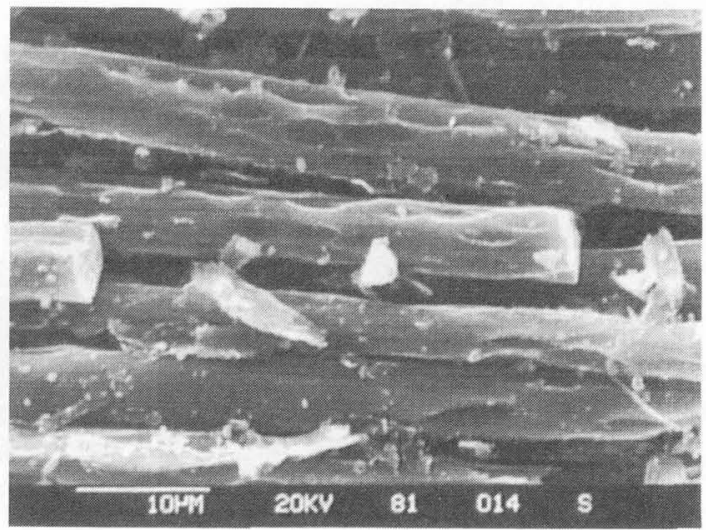

Photo 8

Ponctuellement, nous observons également l'apparition de carbure de silicium (en faible quantité) faisant intervenir l'équilibre suivant :

$$
\mathrm{SiO}_{2}+3 \mathrm{C} \rightleftarrows \mathrm{SiC}+2 \mathrm{CO}
$$

3.2 INFLUENCE DU FER. - La présence de fer sous forme élémentaire ou combinée s'accompagne d'une augmentation de la cinétique d'oxydation des carbones à température élevée [6-8].

Dans nos conditions opératoires, nous constatons une réduction du fer combiné (essentiellement $\mathrm{Fe}_{2} \mathrm{O}_{3}$ ) à l'état élémentaire avec formation de whiskers de fer (diamètre : $3 \mu \mathrm{m}$, longueur environ $50 \mu \mathrm{m}$ ) (Photo 13) qui se déposent dans les zones "froides" de l'appareillage. La teneur en whiskers est d'autant plus élevée que le graphite est moins purifié. 


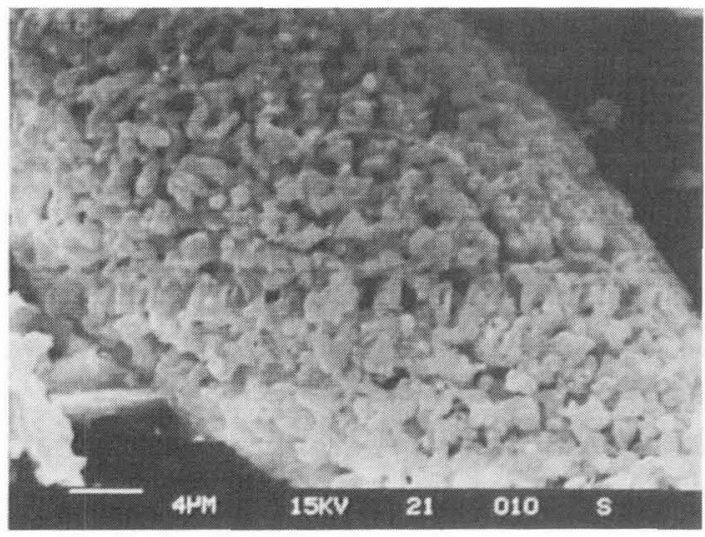

Photo 9

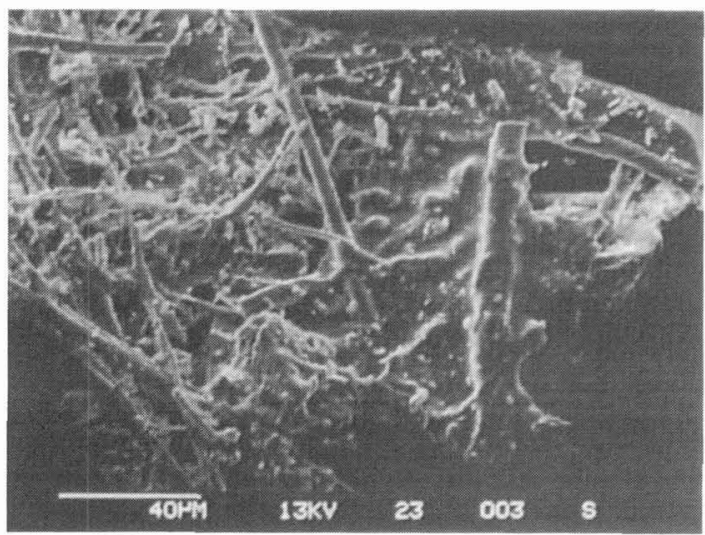

Photo 11

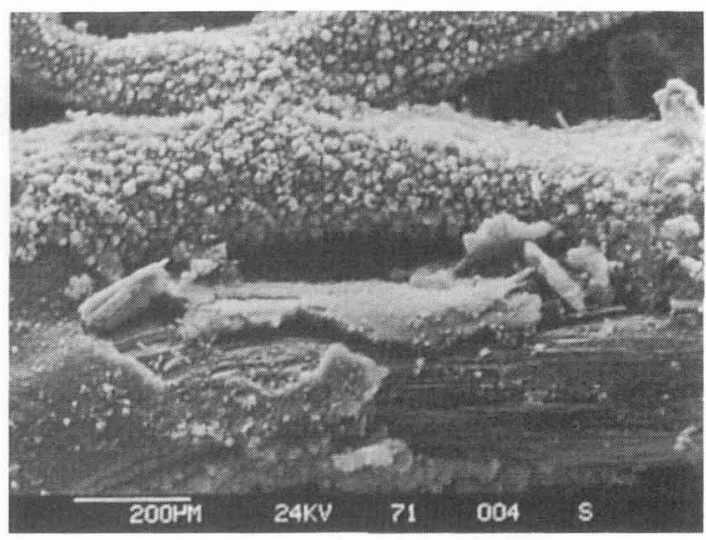

Photo 10

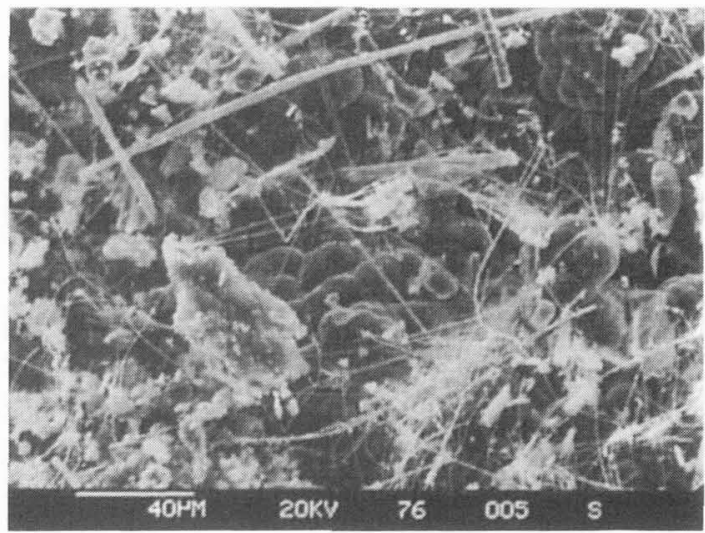

Photo 12

Photo 9. - Squelette d'alumine d'une fibre silico-aluminée.

Photo 10. - Dépôt carboné en petites particules sur les gaines de pyrocarbone.

Photo 11. - Observation du film carboné déposé sur des fragments de fibres siliceuses.

Photo 12. - Fines fibres de silice cristallisée provenant de la réaction (2).

Sur certaines particules de graphite (Photo 14), le fer semble dispersé sur et dans les cristallites de graphite.

3.3 INFLUENCE DU CALCIUM. - L'examen de la section de la spirale de graphite artificiel, au niveau de la cassure (Photo 15), permet de mettre en évidence deux zones de compacité différente :

- interne : dense, contenant du fer comme impureté et disparition du calcium;

- externe : friable, disparition du fer (whiskers) et formation de carbure de calcium.

Pour rendre compte de la migration du calcium vers l'extérieur, nous avons effectué une série d'essais à 600,900 et $1200 \mathrm{~K}$ en déposant des particules de $\mathrm{CaO}$ ou $\mathrm{CaCO}_{3}$ sur des surfaces fraîchement clivées de pyrographite $\operatorname{HOPG}\left({ }^{1}\right)$. A $1200 \mathrm{~K}$, le carbonate est décomposé en $\mathrm{CaO}$, et au voisinage de ces particules apparaissent des piqûres de corrosion

$\left({ }^{1}\right)$ HOPG $=$ Highly Oriented Pyrolitic Graphite : Pyrographite recuit sous pression à $300^{\circ} \mathrm{C}$. 


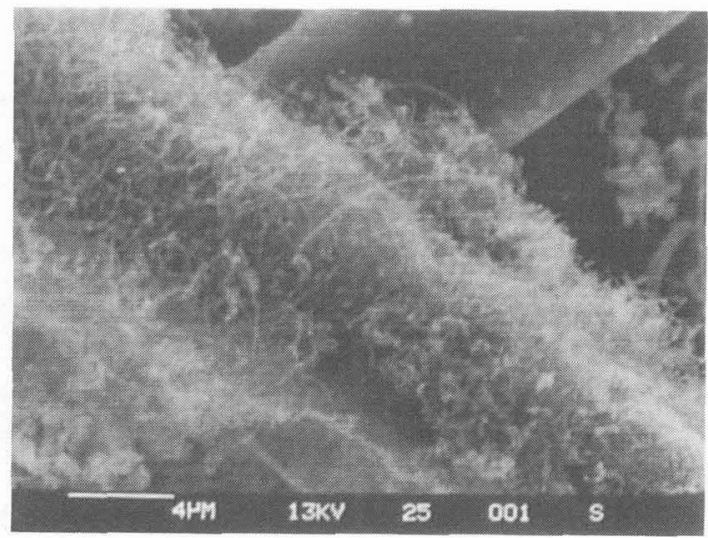

Photo 13

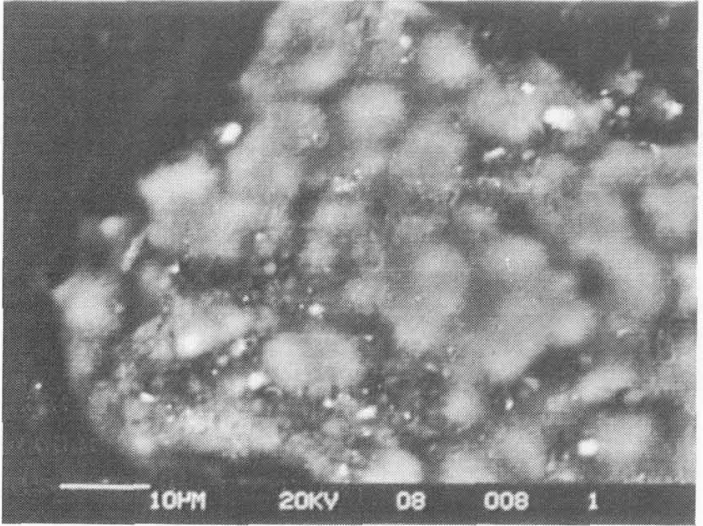

Photo 14

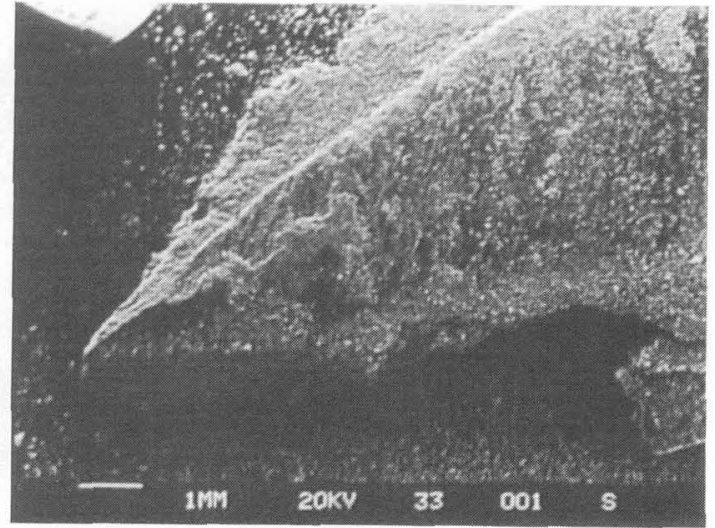

Photo 15

Photo 13. - Whiskers de fer provenant de la réduction des impuretés du graphite.

Photo 14. - Observation au MEB en électrons rétrodiffusés du fer déposé et probablement intercalé entre les plans graphitiques.

Photo 15. - Section de la spirale de la résistance carbonée présentant une zone friable externe (carbure de calcium) et une zone interne compacte (fer).

(Photo 16) de forme lenticulaire quasi-géométrique. L'aspect perturbé des cristallites de graphite (Photo 17) suggère l'intercalation, puis la désorption brutale du réactif entre les feuillets graphitiques. Une réaction similaire peut être observée sur les fibres de carbone (Photo 18) avec l'apparition de particules de carbure de calcium sur l'extrémité des fibres carbonées (Photo 19).

Ces observations nous permettent de supposer qu'à température élevée, le calcium combiné est réduit à l'état métallique, qu'il s'intercale dans le graphite (formation de $\mathrm{CaC}_{6}$ ) et diffuse entre les feuillets graphitiques pour finalement former le carbure $\mathrm{CaC}_{2}$ à température élevée. 


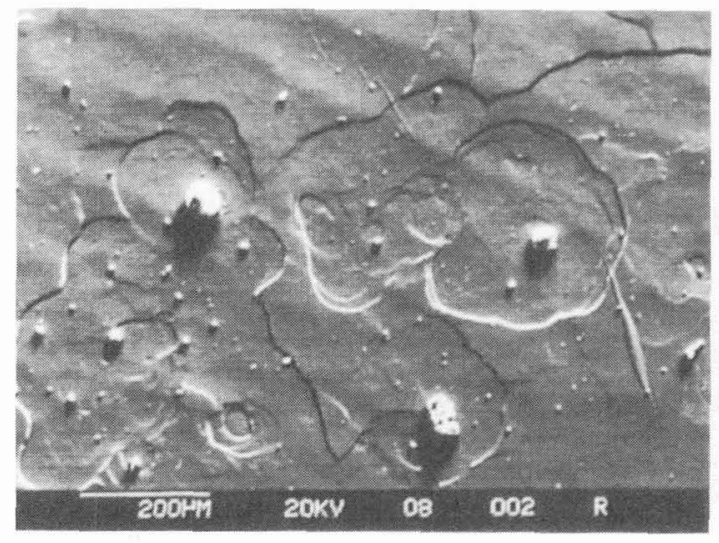

Photo 16

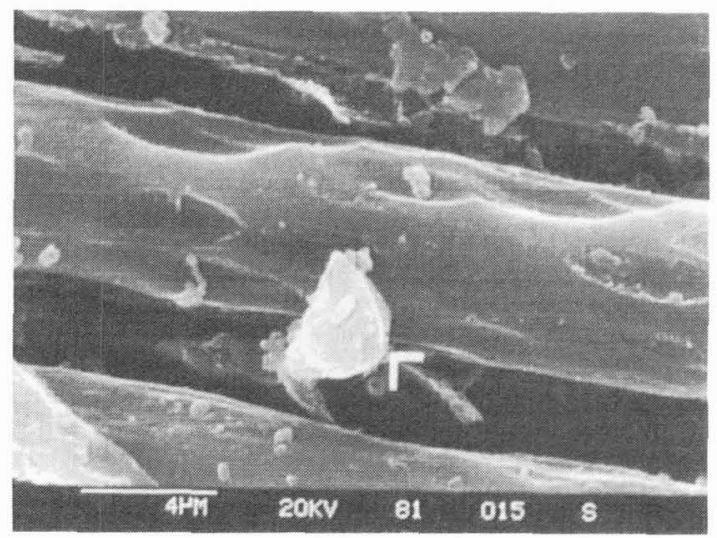

Photo 18

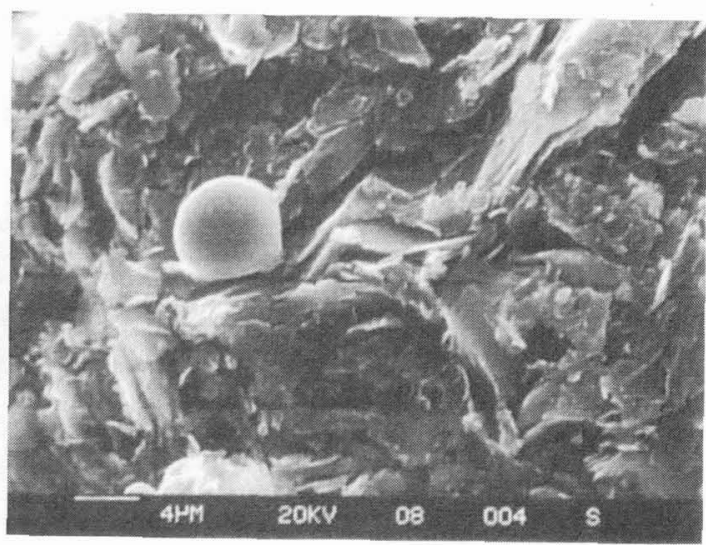

Photo 17

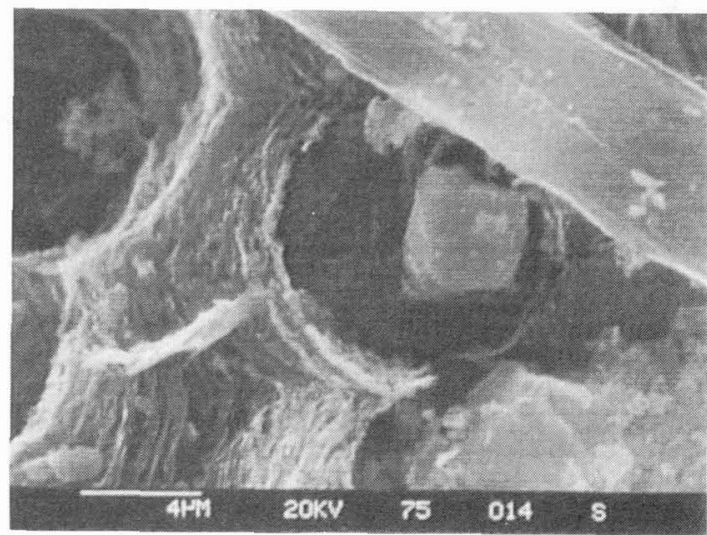

Photo 19

Photo 16. - Piqûres de corrosion sur HOPG apparaissant en présence de carbonate de calcium à $1200 \mathrm{~K}$.

Photo 17. - Feuillets de graphite exfolié en présence de chaux, à la surface du HOPG.

Photo 18. - Présence de carbure de calcium au voisinage des piqûres de corrosion le long des fibres de carbone.

Photo 19. - Présence de carbure de calcium à l'extrémité des fibres de carbone.

\subsection{INFLUENCE DE LA TEXTURE DES MATÉRIAUX COMPOSITES.}

3.4.1 Matériaux $A$ et $B$. - Les matériaux composites $\mathrm{G} / \mathrm{C}$ sont constitués par des fibres exPAN enrobées par une matrice carbonée :

- à base de résine pour former un matériau 2D : produits A et $\mathrm{B}$ (Photo 20) ;

- par dépôt CVD de pyrocarbone pour former un matériau 3D : produit C (Photo 21).

Les matériaux A et B sont constitués de fibres conductrices noyées dans une matrice plus résistante dont la conductivité augmente avec la température de traitement. Les lignes de courant traversant l'échantillon (Fig. 1b) suivent la direction d'un faisceau de fibres. A chaque changement de direction ou arrêt du faisceau de fibres, la température s'élève et le carbone se sublime progressivement jusqu'à disparition de la matrice (Photo 22). En l'absence de contact 


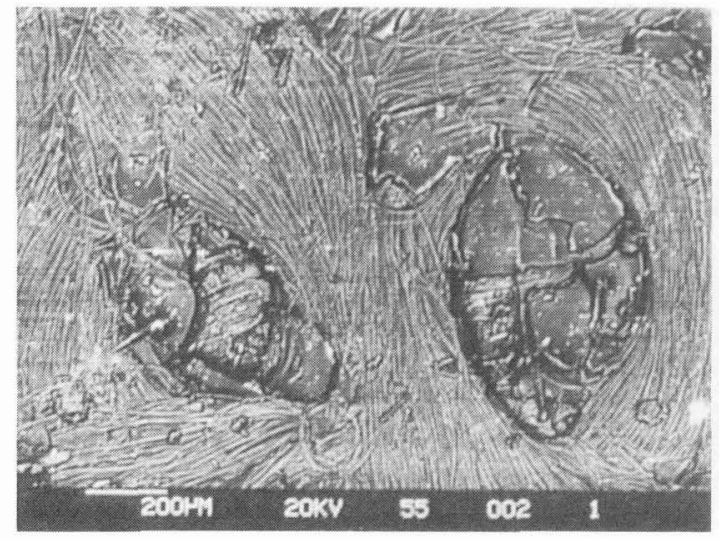

Photo 20

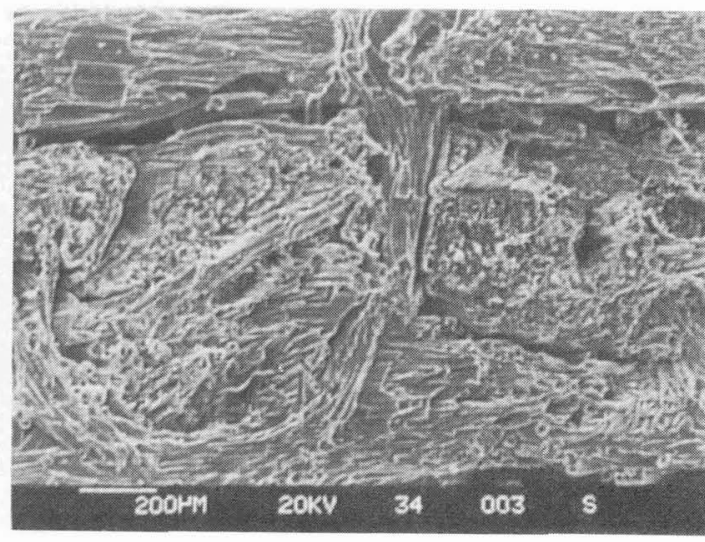

Photo 21

Photo 20. - Surface de matériaux composite $\mathrm{C} / \mathrm{C}$ à matrice ex-résine.

Photo 21. - Surface de matériaux composite $\mathrm{C} / \mathrm{C}$ à matrice pyrocarbone.

direct entre faisceaux de fibres, un arc électrique apparaît provoquant un amincissement des fibres; leurs extrémités présentent alors un aspect acéré (Photo 23). Etant donné le maillage croisé des fibres de carbone, l'arcage électrique s'effectue en marches d'escalier (Photo 24). Les propriétés mécaniques du matériau diminuent très rapidement jusqu'à la rupture finale avec cassure nette des fibres amincies (Photo 22).

Pour améliorer leur comportement, les composites $\mathrm{C} / \mathrm{C}$ doivent présenter une conductivité équivalente pour les fibres et la matrice et donc être graphités. La température de fabrication des composites $\mathrm{C} / \mathrm{C}$ imprégnés par de la résine n'est pas un facteur suffisant : la durée de vie du produit $B\left(2000^{\circ} \mathrm{C}\right)$ n'est que légèrement améliorée par rapport au produit $A\left(1000^{\circ} \mathrm{C}\right)$. Par contre, le matériau B épuré, traité vers $2700^{\circ} \mathrm{C}$, présente un faible taux d'impureté et une bonne conductivité électrique pour les fibres comme pour la matrice. Il conserve ses propriétés mécaniques après environ $5000 \mathrm{~h}$ d'utilisation.

3.4.2 Matériaux C. - Dans ces matériaux, la matrice est constituée d'un dépôt CVD de pyrocarbone. Les fibres de carbone sont enrobées par des plans graphitiques, le passage du courant s'effectue en partie selon la direction perpendiculaire à ces plans (conductivité électrique inférieure) ; on retrouve alors le mécanisme de corrosion décrit ci-dessus. Les produits $\mathrm{C}$ standards ont ainsi une durée de vie de 3000 à $5000 \mathrm{~h}$. L'utilisation d'un dépôt de pyrocarbone $(20 \mu \mathrm{m})$ permet d'augmenter considérablement la durée d'utilisation qui peut dépasser $8000 \mathrm{~h}$.

Par contre, un dépôt superficiel de SiC d'épaisseur comparable, n'apporte pas d'amélioration sensible par rapport au matériau standard (présence de fissures dues aux coefficients de dilatation différents du composite $\mathrm{C} / \mathrm{C}$ et du $\mathrm{SiC}$ ).

\section{Conclusion.}

L'utilisation des résistances carbonées à haut flux est maintenant du domaine de la réalité, en prenant les précautions suivantes :

- absence de silice, de fer et de calcium sous forme élémentaire ou combinée ; 


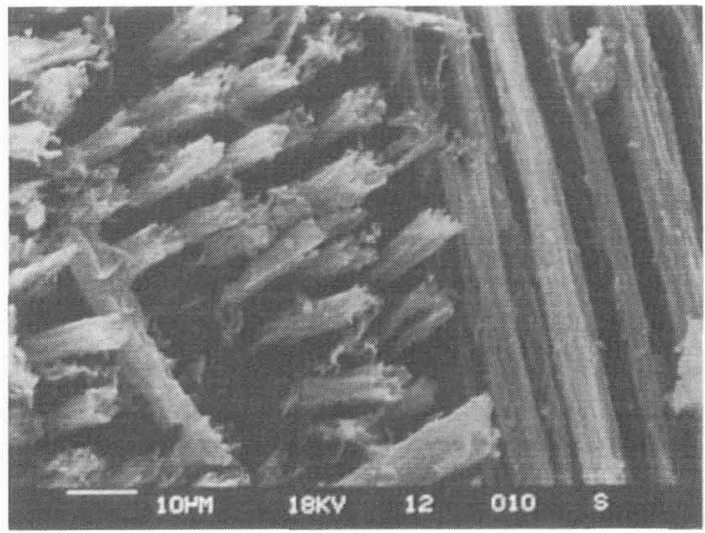

Photo 22

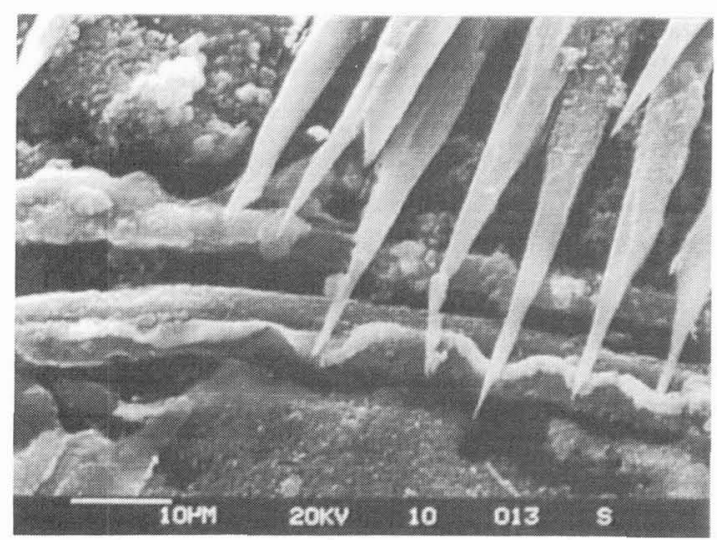

Photo 23

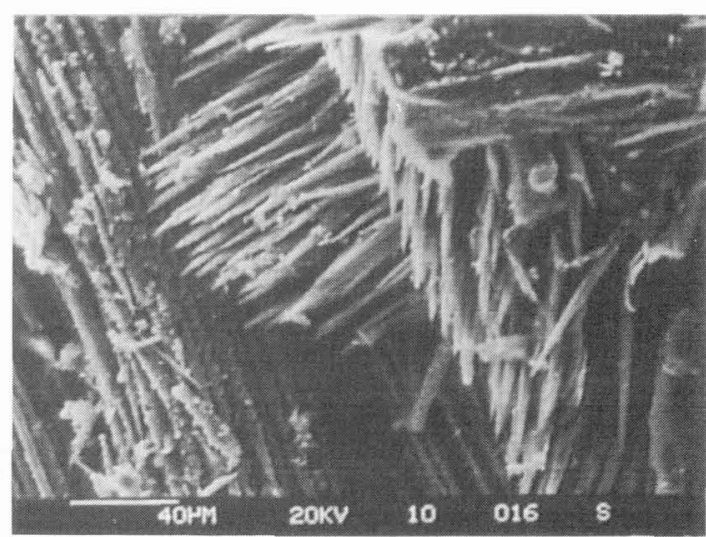

Photo 24

Photo 22. - Disparition de la matrice carbonée entre les fibres et corrosion de celles-ci.

Photo 23. - Mise en évidence d'un arcage électrique entre faisceaux de fibres dégagées de la matrice et apparition de "marches d'escalier".

Photo 24. - Mise en évidence de l'apparition de "marches d'escalier".

- utilisation de matériaux carbonés très bien graphités (matrice carbonée isotrope, dépôt de pyrographite superficiel suffisamment épais : $20 \mu \mathrm{m}$ au minimum).

\section{Bibliographie}

[1] SOHN K.Y., LEE J.Y., Proc. 20th Carb. Conf. (1991 p. 338.

[2] ISMAIl I.M.K., MAHOWAld M.A., Proc. 20th Carb. Conf. (1991) p. 366.

[3] Montaudon M., Gery P., Ghristin F., Proc. 20th Carb. Conf. (1991) p. 384.

[4] Fernandez J.J., Bermejo J., Fuente E., Romero E., Menendez R., Proc. 20th Carb. 
Conf. (1991) p. 346.

[5] FitZer E., Carbon (1978) 3.

[6] Les Carbones par le Groupe Français d'Etude des Carbones, Masson Ed. (1964) pp. 277-385.

[7] Mukai K., Ohkubo S., Orita M., Fujimoto K., Fujimoto K.I., Proc. 20th Carb. Conf. (1991) p. 562.

[8] Bi X.X., Ecklund P.G., Stencel J.M., Taulbee D.N., Ni H.F., Derbyshire F.J., ENDO M., Proc. 20th Carb. Conf. (1991) p. 518. 